\title{
Nanoscale hyperspectral imaging of amyloid secondary structures in
} liquid

\section{Journal Article}

\section{Author(s):}

Lipiec, Ewelina; Kaderli, Janina; Kobierski, Jan; Riek, Roland; Skirlinska-Nosek, Katarzyna; Sofinska, Kamila; Szymonski, Marke; Zenobi, Renato (iD)

Publication date:

2021-02-23

Permanent link:

https://doi.org/10.3929/ethz-b-000441973

Rights / license:

In Copyright - Non-Commercial Use Permitted

Originally published in:

Angewandte Chemie. International Edition 60(9), https://doi.org/10.1002/anie.202010331 
Nanoscale hyperspectral imaging of amyloid secondary structures in liquid

Ewelina Lipiec ${ }^{1,2}$, Janina Kaderli ${ }^{2}$, Jan Kobierski ${ }^{3}$, Roland Riek ${ }^{2}$, Katarzyna Skirlińska-Nosek ${ }^{1}$, Kamila Sofińska ${ }^{1}$, Marek Szymoński ${ }^{1}$, and Renato Zenobi ${ }^{*}$

${ }^{1}$ M. Smoluchowski Institute of Physics, Jagiellonian University, Lojasiewicza 11, 30-348 Kraków, Poland

${ }^{2}$ Department of Chemistry and Applied Biosciences, ETH Zurich, 8093 Zurich, Switzerland

${ }^{3}$ Department of Pharmaceutical Biophysics, Faculty of Pharmacy, Jagiellonian University Medical College, 31-007 Kraków, Poland

\begin{abstract}
Abnormal aggregation of amyloid- $\beta$ is a very complex and heterogeneous process. Due to methodological limitations, the aggregation pathway is still not fully understood. In this communication a new approach is presented in which the secondary structure of single amyloid- $\beta$ aggregates is investigated with tip-enhanced Raman spectroscopy (TERS) in a liquid environment. Clearly resolved TERS signatures of the amide I and amide III bands enabled a detailed analysis of the molecular structure of single aggregates at each phase of the primary aggregation of amyloid- $\beta$ and also of small species on the surface of fibrils attributed to secondary nucleation. Notably, a $\beta$-sheet rearrangement from antiparallel in protofibrils to parallel in fibrils is observed. This study allows better understanding of Alzheimer's disease etiology and the methodology can be applied in studies of other neurodegenerative disorders.
\end{abstract}

The secondary structure and conformational transitions of peptides and proteins determine their biological functionality and the ability to interact with other biomolecules. On the other hand, structural rearrangements are also a hallmark of abnormal protein aggregation, one of the key events in the neurodegenerative processes associated with dementia. ${ }^{[1,2]}$ Recently the application of molecular nano-spectroscopy including TERS and infrared nano-spectroscopy brought new insights into research on amyloids. TER spectroscopy established that the individual fibrils of $\beta 2$ microglobulin exhibit a predominant $\beta$-sheet structure ${ }^{[3]}$ and proved the heterogeneity of human islet amyloid fibrils. ${ }^{[4]}$ TERS also gave insight into the secondary structure of insulin ${ }^{[5]}$ or amyloid- $\beta\left(\mathrm{A} \beta_{1-42}\right)^{[6,7]}$ aggregates as well as the insulin aggregates influenced by several aggregation inhibitors during aggregation. ${ }^{[8]}$ Simultaneously, infrared nano-spectroscopy has been increasingly used for the nanoscale investigation of amyloids such as Josephin, ${ }^{[9]}$ insulin, ${ }^{[10]}$ and to study the interaction between $A \beta_{1-42}$ and lipids. ${ }^{[11]}$

The secondary structure of delicate biological samples, including amyloids, should be studied under physiological conditions (i.e., in solvent), because upon drying they may change their conformation, which is essential for their stability and biochemical activity. ${ }^{[12]}$ However, to the best of our knowledge, previous nano-spectroscopic studies of amyloids were all performed in 
dry conditions, with a single exception: Centrone et al. reported infrared spectra and singlefrequency maps of the core recognition module of $A \beta$ peptide with a vertical resolution of a few hundreds of nanometers. ${ }^{[13]}$

Here, we report on a study in which hyperspectral mapping of individual various $A \beta$ forms at subsequent stages of the abnormal aggregation, precisely fibrillation, including young globular oligomers, immature granular fibrils and smooth mature fibrils (generally called aggregates) in the native liquid environment with nanometer spatial resolution is performed.

To fully explore the TER signature of amyloid- $\beta$ in the liquid environment, and get deep insights into the spectral changes related to ongoing aggregation we extract TERS band characteristics for amyloid aggregates at various phases of the aggregation process. A multivariate data analysis was applied to treat the TERS data acquired from various types of aggregates. All the measured aggregates were located close to each other on the same gold surface. To find a representative time point of $A \beta_{1-42}$ aggregation in order to follow the secondary structure of $A \beta_{1-42}$ aggregates at all phases of the aggregation process (including oligomers, protofibrils and mature fibrils), we performed a comprehensive study of the timedependent aggregation (Supporting Information). The incubation time of $A \beta_{1-42}$ (at $37^{\circ} \mathrm{C}$ ) was selected to monitor the aggregation process before full fibrillation. ${ }^{[7]}$ Details on the samples preparation procedure and data processing are provided in Supporting Information. To reduce the dataset dimensionality and establish relationships between variables, Hierarchical Cluster Analysis (HCA) using Ward's method was performed on 119 spectra randomly acquired at various locations of 10 different samples with 10 different TERS probes.

The $A \beta$ aggregation process is complex and contains several secondary structures. ${ }^{[14]} \mathrm{With}$ the help of TERS they can in principle be localized to the single species level. Indeed, the analysis revealed the presence of three groups of spectra. The dendrogram and the averaged spectra for each cluster are presented in Figure 1 (A-C). All the bands expected in TER and Raman spectra of proteins and peptides including the amide I mode in the spectral range $1700-1600 \mathrm{~cm}^{-1}$, the amide II from 1580 to $1500 \mathrm{~cm}^{-1}$, the $\mathrm{CH}_{3}, \mathrm{CH}_{2}$ scissoring motions at $\sim 1450$, the $\mathrm{C} \alpha-\mathrm{H} / \mathrm{N}-\mathrm{H}$ at $1390 \mathrm{~cm}^{-1}$, the amide III from 1340 to $1200 \mathrm{~cm}^{-1}$, the Phe ring breathing mode at $1004 \mathrm{~cm}^{-1}$, and the signatures from amino acid residues are present in each averaged spectrum. Detailed band assignments are provided in Supporting Information, see Table S1. Supplementary Table S1 also presents a comparison of the band spectral positions visible in Raman spectra, AFMTERS, and STM-TERS data acquired in ambient conditions. In the three averaged spectra presented in Figure 1, the amide band positions and intensities were found to vary, which 
indicates differences in peptide secondary structure. The band assigned to $\beta$-turns/disordered coils or $\beta$-hairpin ${ }^{[15]}$ at $\sim 1650 \mathrm{~cm}^{-1}$ characteristic for immature aggregates of amyloid- $\beta$ containing antiparallel $\beta$-sheet structure is present in the green and red spectra. Interestingly, bands characteristic for the Tyr and Phe amino acid residues at $1611 \mathrm{~cm}^{-1}$ only appear in the green spectra. The relatively high intensity of this band can be considered as a hallmark of young aggregates, i.e., oligomers. ${ }^{[16]}$ During the ongoing aggregation process, this band decreases due to a change of the local environment of the Tyr and Phe amino acids. ${ }^{[16]}$

We thus attribute the green spectrum to the earliest aggregation intermediates such as oligomers. We interpret the red spectrum to represent more advanced stages of the aggregation, for example, the protofibrillar stage. A decrease of the intensity of the band associated with $\beta$ turns/disordered coils or $\beta$-hairpin at $1650 \mathrm{~cm}^{-1}$ is detected only in the yellow spectrum, which, together with a relatively high intensity of the band attributed to parallel $\beta$-sheet at $1626 \mathrm{~cm}^{-1}$ indicates the presence of a more ordered structure, typical for mature fibrils displaying the cross- $\beta$ structure with $\beta$-sheets oriented parallel. ${ }^{[17,18]}$ Such an arrangement of amyloid $\beta_{1-42}$ fibrils was confirmed by other techniques such as cryo-electron microscopy ${ }^{[19]}$ and solid-state NMR spectroscopy combined with mass-per-length EM measurements, ${ }^{[20]}$ which yielded the fibril structure with atomic resolution. Additionally the high-frequency component characteristic for antiparallel $\beta$-sheets appearing as a shoulder at $\sim 1684 \mathrm{~cm}^{-1}$ in the green and red spectra, according to the interpretation of infrared spectra for amyloid aggregation, ${ }^{[21]}$ is not present in the yellow spectrum assigned to fibrils. The $1695 \mathrm{~cm}^{-1}$ band, attributed to extended $\beta$-strands and $\beta$-sheets or turns, vanishes from the green spectrum through the red to the yellow one, confirming that the peptide reorganizes its $\beta$-strands from an antiparallel to a parallel $\beta$ sheet. A significant shift of the amide III to higher wavenumbers observed in the yellow spectrum in comparison to the green and red ones also confirms the rearrangement of $\beta$-sheets from antiparallel to parallel orientation. These spectral differences were observed in the individual TER spectra acquired from fibrils, protofibrils and oligomers incubated for 24 (Figure 2) and 36 hours (Figure S3).

Remarkably, the TER spectra acquired in liquid give better reproducibility and higher signalto-noise ratio (SNR) than the TERS data collected in dry conditions. The main spectral difference is the clearly resolved TERS signature of the amide I band, which is mainly absent in TERS and SERS data acquired in air. To verify whether the presence of the liquid influences the Raman cross-section for the amide I band, DFT calculations were carried out to simulate the spectra of the Ala-Phe dipeptide in vacuum, Ala-Phe in the presence of two explicit water 
molecules, and Ala-Phe in the presence of water as a solvent. No significant changes of the amide I band intensity were observed in the theoretical spectra, see the Supporting Information. Recently it has been shown that the absence of the amide I band in TER and SER spectra is mainly due to plasmon-induced dissociation of the peptide backbone. ${ }^{[22]}$ However, the increasing temperature triggers charge driven reactions because the probability of electronic transition from a vibrationally excited state (thermal excitation) is higher than from the ground state. Due to the liquid medium, heat dissipation from the tip is more efficient, which results in a lower effective temperature of the plasmonic structure of the surface, ${ }^{[23]}$ which impedes plasmon-induced reactions. This effect increases the stability of enhanced Raman signal, in agreement with our results. More details can be found in Supporting Information. Thus, it may be the electron and radical scavenging properties and/or the high heat capacity of water that prevents decomposition of the sample, such that the amide I band can still be observed in all amyloid- $\beta$ spectra, see Supporting Information. This provides an additional important motivation to record TERS in the liquid environment when studying the secondary structure of proteins and peptides at the nanoscale.

The AFM topography acquired in liquid together with cross-sections through oligomers (green lines), granular protofibrils (red lines) and smooth fibrils (yellow lines) is presented in Figure 1D and E, respectively. The height cross-section profiles (Figure $1 \mathrm{E}$ ) clearly indicate the morphological differences among species assigned to particular groups: fibrils (profile 1-3), protofibrils (profile 4-6), and oligomers (profiles 7-9). A detailed description of AFM height profiles is provided in Supporting Information. 


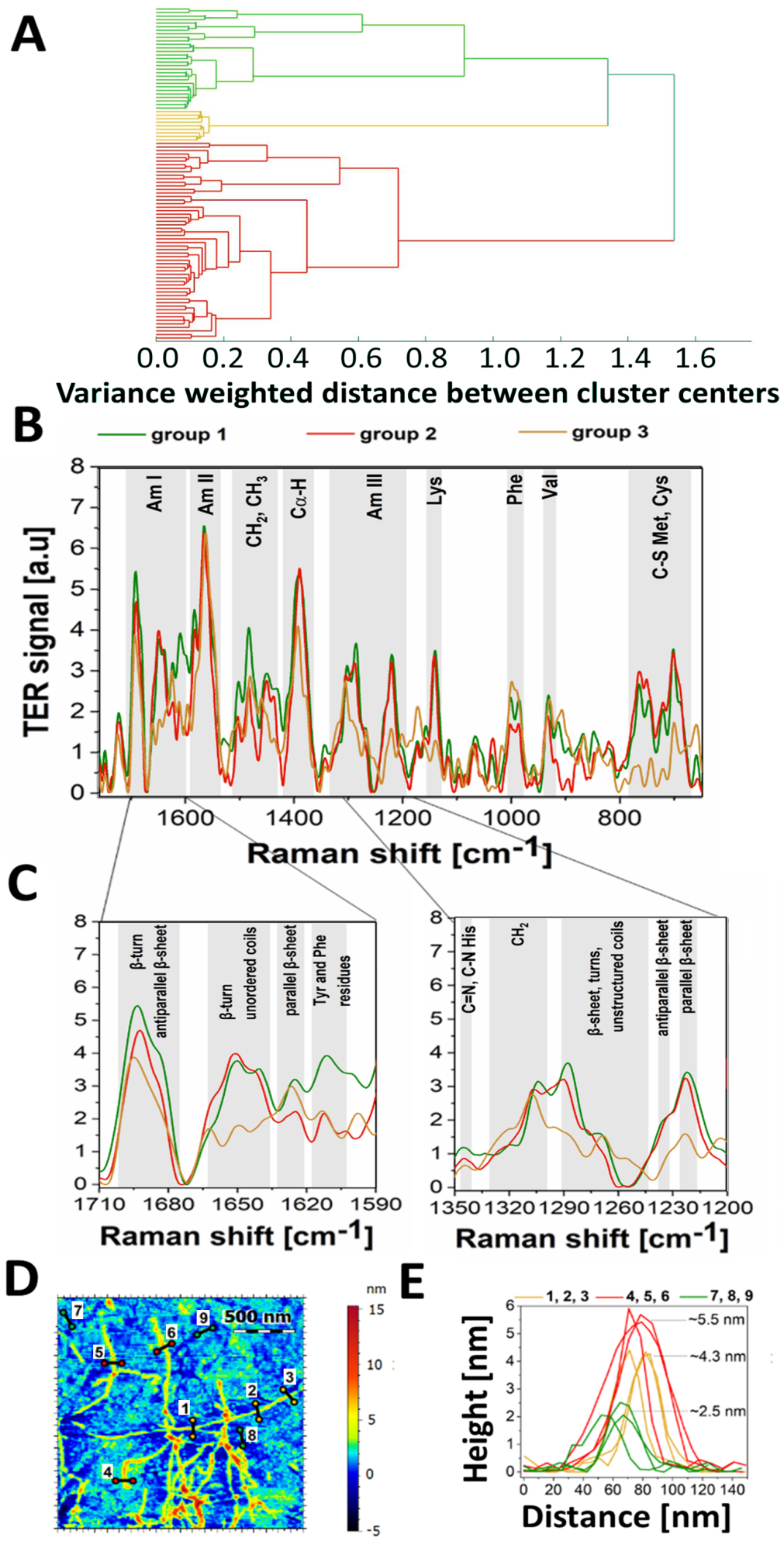

Figure 1. TER signature of amyloid- $\beta$ in liquid. A) The results of HCA analysis for $\sim 100$ randomly chosen TER spectra showing the classification into three groups of spectra attributed to oligomers (green), protofibrils (red) and fibrils (yellow) and B) averaged TER spectra for each of these groups with $C$ ) the magnification of the amide I and amide III regions. D) AFM topography image of $A \beta_{1-42}$ deposited on a gold substrate (image obtained using tapping mode in liquid) with E) height profiles across all three aggregated forms; the incubation time was 24 hours. 
Figure 2 shows individual TER spectra acquired from $A \beta_{1-42}$ sample incubated for 24 hours and then deposited onto a gold substrate, at various locations highlighted in each AFM scan. The raw and smoothed spectrum acquired from oligomer and neighbouring place free of aggregates is shown in Figure 2 A to demonstrate typical SNR of the analysed data. The spectra collected from oligomers (Figure $2 \mathrm{~B}$, green) exhibit Raman signatures typical for immature aggregates containing also partially native secondary structure, which is confirmed by the presence of a relatively intense band from Tyr and Phe amino acid residues. The spectral signature of the amide I attributed to antiparallel $\beta$-sheet structure was detected in spectra acquired from oligomers (green) and protofibrils (red). The yellow spectra collected from smooth fibrils indicate mainly a parallel $\beta$-sheet orientation. Analogical results for $A \beta_{1-42}$ after 36 hours of aggregation are provided in Supporting Information. The acquired spectra confirmed that the secondary structure of oligomers, fibrils and protofibrils do not change with the increase of the aggregation time. However, after 36 hours of incubation the ratio of subsequent aggregates changes, the number of protofibrils is less abundant in comparison to fibrils.

Despite the high heterogeneity of the samples, which is mainly responsible for the variability of the spectra, the relative content of bands related to the particular secondary structure (see Table S1) is characteristic for each group of aggregates. Figure S2 shows a HCA analysis of the spectra acquired from particular aggregates, demonstrating $87 \%$ correct classification to the corresponding groups. Only two spectra of fibrils were misclassified as protofibrils. It is worth noting that the whole surface of the sample is likely covered by amyloid monomers and small aggregates such as dimers, trimers etc. (see Figure 2 A). The signal from such aggregates may contribute to the acquired spectra depending on the spatial resolution, pixel size, and precise tip position with respect to particular aggregates. The variability of the TERS band intensities ${ }^{[24]}$ observed in Figure 2 may be caused by various orientations of the measured molecules with respect to the electromagnetic field under the tip, which is along the tip axis and thus perpendicular to the metallic substrate. 


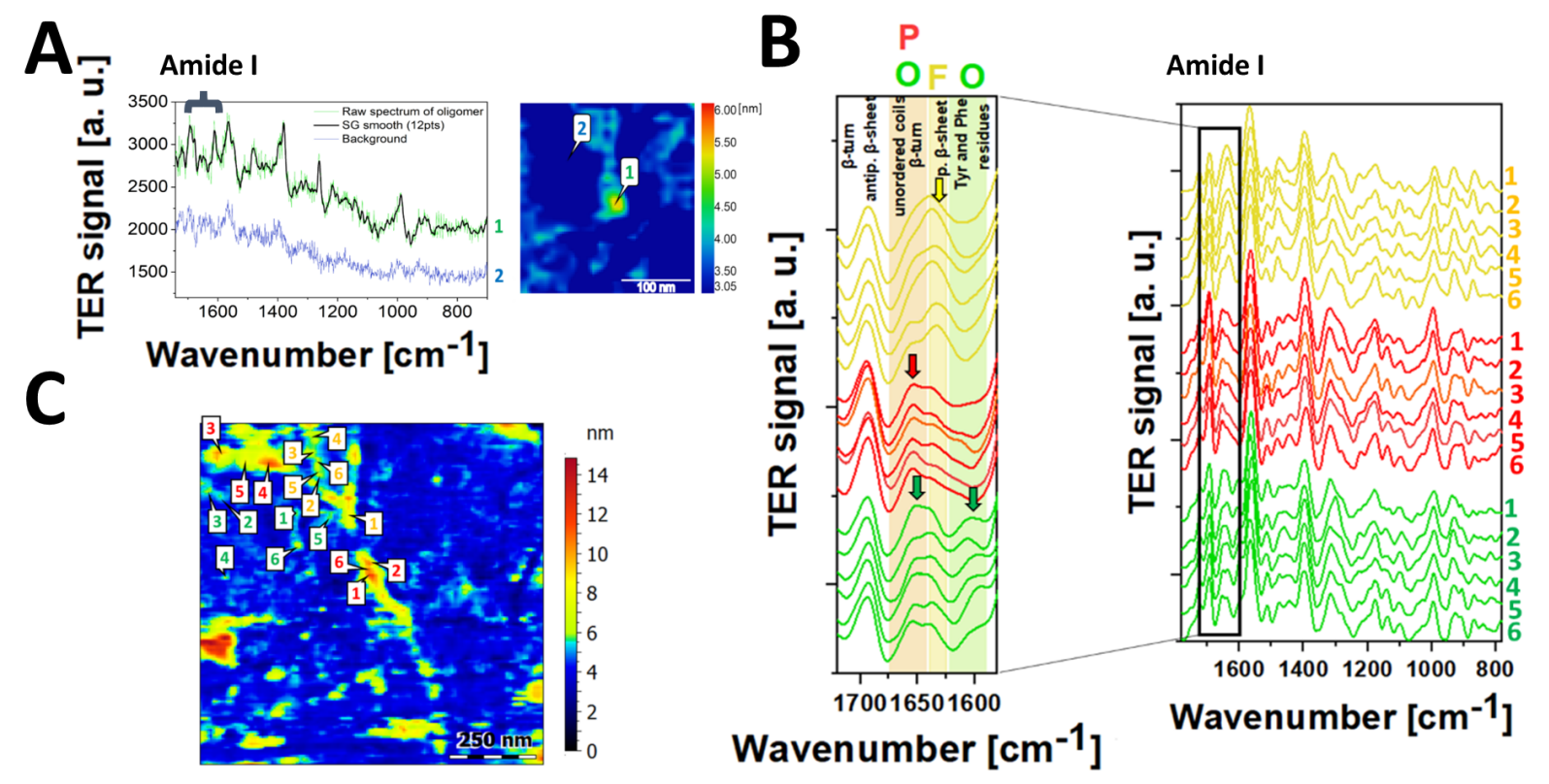

Figure 2. Individual TER spectra of $\left.\mathbf{A} \boldsymbol{\beta}_{1-42} ; A\right)$ left: raw TER spectrum of oligomer (green), smoothed spectrum of oligomer (black) and TER spectrum acquired from the neighbouring place free from $A \beta_{1-42}$ aggregates (blue); right: the AFM topography with the marked places of the spectra acquisition.; B) TER spectra acquired from fibrils (yellow), protofibrils (red) and oligomers (green) with the locations of the spectra acquisition (C); the incubation time was 24 hours.

The conformational rearrangement of $\beta$-sheets from antiparallel orientation in oligomers to parallel in fibrils was already observed for $A \beta$ via Raman ${ }^{[16]}$ and infrared ${ }^{[25-27]}$ spectroscopies or $\mathrm{NMR}^{[28]}$. However, it has not been detected at the nanoscale yet. Moreover, we observed bands characteristic for antiparallel orientation of $\beta$-sheets in the spectra acquired from single segments of protofibrils, suggesting that $\beta$-sheets may arrange parallel during the transformation from protofibrils to fibrils. The loss of the antiparallel $\beta$-sheet signature in the amide I region during amyloid- $\beta$ aggregation has been detected only with FTIR spectroscopy so far, but the acquired signal was averaged from bulk samples. ${ }^{[21]}$ Due to methodological limitations, such structural rearrangement upon the conversion from protofibrils to fibrils has never been observed directly so far. Fractionation of protofibrils from heterogenous bulk samples might not be sufficient due to similar mass of protofibrils and fibrils, which has limited the investigation of the secondary structure of separated protofilbrillar aggregates.

TERS maps in Figure 3 show the distribution of spectra attributed to oligomers, protofibrils and fibrils containing mainly unstructured, antiparallel $\beta$-sheet and parallel $\beta$-sheet secondary structures, respectively. Representative results are given in Figure 3, which presents the values of the Pearson correlation coefficient between derivatives (to avoid baseline influence) of the marker TER spectra described above and from each spectrum of the map. Figure 3 confirms 
the spatial distribution postulated earlier of disordered structures (green spectra), which are distributed randomly on the sample surface in contrast to the antiparallel $\beta$-sheets (red) and parallel $\beta$-sheets (yellow) located mainly on protofibrils and fibrils. Additionally, the TERS maps demonstrate that small aggregates with anti-parallel $\beta$-sheet structure are in the vicinity, or possibly attached to the surface of the protofibrils and fibrils (Figure 3). We relate the presence of these species to the ongoing secondary nucleation process on the surface of fibrils and protofibrils. ${ }^{[29]}$ Based on detailed kinetic measurements it was demonstrated that on the surface of fibrils of $A \beta_{1-42}$ oligomers are formed, which serve as new seeds for aggregation. This mechanism of secondary nucleation is thus most powerful and the driving force of fibril aggregation of $A \beta_{1-42}$ at least under the conditions studied. ${ }^{[29]}$ Only recently, ultrastructural evidence for the existence of time-intermediate growing aggregates along the sides of fibrils was observed using cryo-electron microscopy (EM) and special conditions. ${ }^{[30]}$ The TERS studies strengthen these findings and further highlight that these surface-attached entities are composed of anti-parallel $\beta$-sheet secondary structures as the primary seeds. A cross-section showing the anti-parallel $\beta$-sheet distribution averaged along the marked part of the fibril is included in Figure 3. We found that the concentration of the small nucleated species drops with distance to the fibril. Second derivatives of marker TER spectra of oligomers, protofibrils and fibrils presented in Figure 1 in comparison with highly correlated spectra extracted from TER map (Figure 3) are shown in the Supporting Information. 

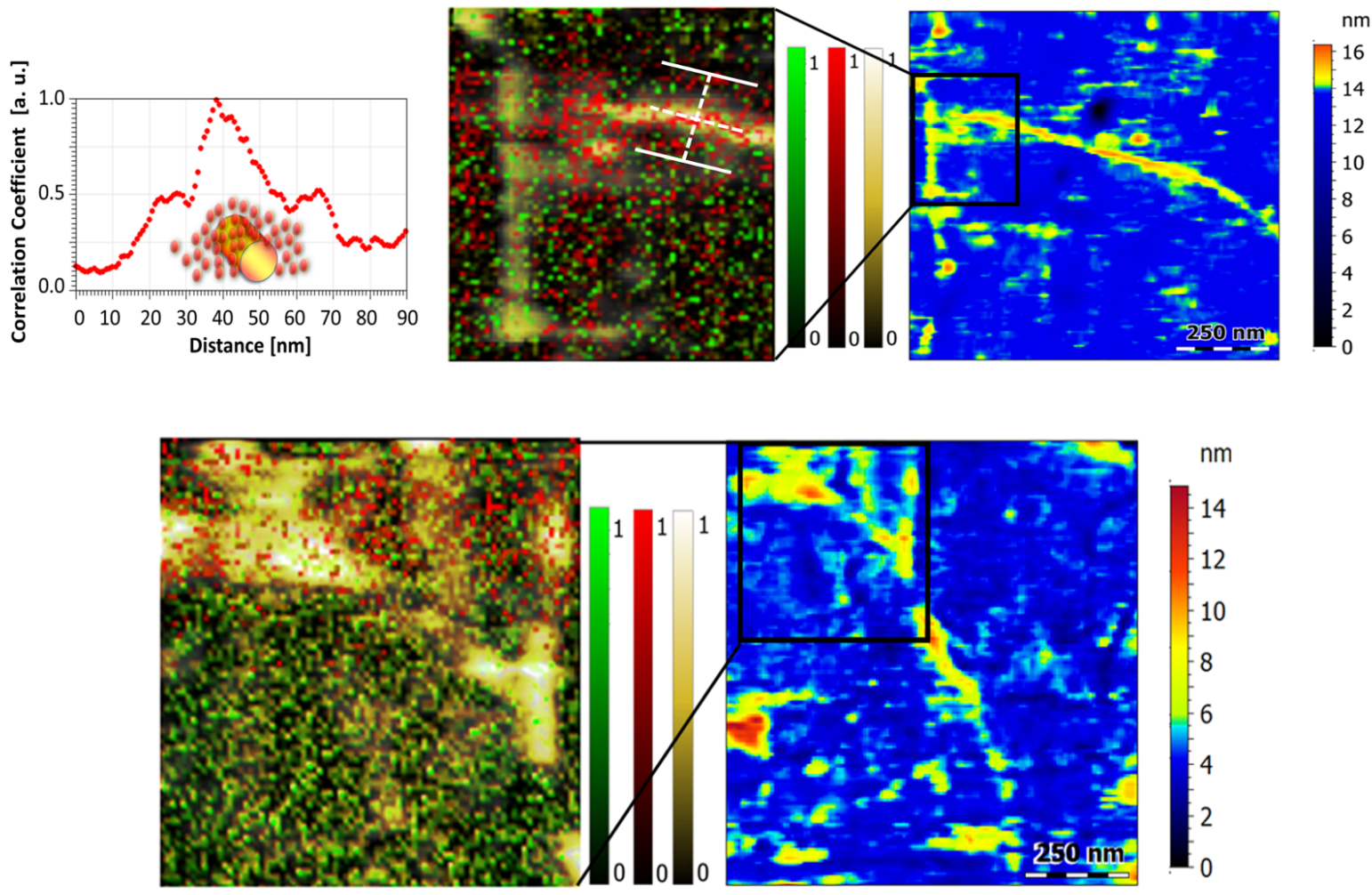

Figure 3 The distribution of TER spectra indicating the presence of mainly unstructured coils/turns (green), antiparallel $\beta$-sheet (red) an parallel $\beta$-sheet (yellow) secondary structures in two representative samples, containing various $A \beta_{1-42}$ aggregates; each pixel displays a normalised value of the Pearson correlation coefficient between one of the three marker TER spectra presented in Figure 1 and the TER spectrum acquired at the particular pixel location. The distributions of these correlation coefficients are superimposed on the AFM topography images. A cross-section through the anti-parallel $\beta$-sheet distribution averaged along the marked part of the fibril is shown. An overview AFM topography of each sample is also presented.

TERS hyperspectral mapping in liquid proved to be efficient to directly follow the amyloid- $\beta$ aggregate structure at each phase of aggregation including small species involved in the secondary nucleation. The methodology applied here enabled us to resolve the amide I band, which was previously impossible with TERS in air. We have also provided direct evidence for the structural arrangement of protofibrils. Our results suggest that the transition from an antiparallel $\beta$-sheet secondary structure to a parallel $\beta$-sheet arrangement may occur during the conversion of protofibrils to fibrils.

\section{Acknowledgments}

E.L was partially supported by a grant from the National Science Center of the Republic of Poland (Narodowe Centrum Nauki-NCN), Grant No. 2014/13/D/NZ1/01014. The data reported 
in this paper are available in the Supporting Information and also in a curated data archive at ETH Zurich (https://www.research-collection.ethz.ch) under the DOI 10.3929/ethz-b000428946. The authors would like to thank Dr Jacek Szczerbiński, Prof. Jakub Barbasz and M.Sc. Eng. Igor Arkov for fruitful discussions.

\section{Conflict of Interest}

The authors declare no conflict of interest.

\section{Keywords}

amyloid- $\beta \bullet$ aggregation pathway $\bullet$ tip-enhanced Raman spectroscopy (TERS)

[1] R. Riek, D. S. Eisenberg, Nature 2016, 539, 227-235.

[2] T. P. J. Knowles, M. Vendruscolo, C. M. Dobson, Nat. Rev. Mol. Cell Biol. 2014, 15, 384-396.

[3] P. Hermann, H. Fabian, D. Naumann, A. Hermelink, J. Phys. Chem. C 2011, 115, 24512-24520.

[4] C. C. Vandenakker, T. Deckert-Gaudig, M. Schleeger, K. P. Velikov, V. Deckert, M. Bonn, G. H. Koenderink, 2015, DOI 10.1002/smll.201500562.

[5] D. Kurouski, T. Deckert-Gaudig, V. Deckert, I. K. Lednev, J. Am. Chem. Soc. 2012, 134, 13323-13329.

[6] S. Bonhommeau, D. Talaga, J. Hunel, C. Cullin, S. Lecomte, Angew. Chemie Int. Ed. 2017, 56, 1771-1774.

[7] E. Lipiec, D. Perez-Guaita, J. Kaderli, B. R. Wood, R. Zenobi, Angew. Chemie - Int. Ed. 2018, 57, DOI 10.1002/anie.201803234.

[8] T. Deckert-Gaudig, V. Deckert, Sci. Rep. 2016, 6, DOI 10.1038/srep39622.

[9] F. S. Ruggeri, G. Longo, S. Faggiano, E. Lipiec, A. Pastore, G. Dietler, Nat. Commun. 2015, 6, 1-9.

[10] S. Rizevsky, D. Kurouski, ChemBioChem 2020, 21, 481-485.

[11] S. Henry, N. B. Bercu, C. Bobo, C. Cullin, M. Molinari, S. Lecomte, Nanoscale 2018, 10, 936-940.

[12] G. Maurstad, M. Prass, L. C. Serpell, P. Sikorski, Eur. Biophys. J. 2009, 38, 11351140 .

[13] G. Ramer, F. S. Ruggeri, A. Levin, T. P. J. Knowles, A. Centrone, ACS Nano 2018, 12, 6612-6619.

[14] E. Lipiec, D. Perez-Guaita, J. Kaderli, B. R. Wood, R. Zenobi, Angew. Chemie Int. Ed. 2018, 57, 8519-8524.

[15] A. W. Smith, J. Lessing, Z. Ganim, C. S. Peng, A. Tokmakoff, S. Roy, T. L. C. Jansen, 
J. Knoester, J. Phys. Chem. B 2010, 114, 10913-10924.

[16] D. Kurouski, R. P. Van Duyne, I. K. Lednev, Analyst 2015, 140, 4967-4980.

[17] L. A. Popova, R. Kodali, R. Wetzel, I. K. Lednev, J. Am. Chem. Soc. 2010, 132, 63246328.

[18] D. Kurouski, M. Sorci, T. Postiglione, G. Belfort, I. K. Lednev, Biotechnol. Prog. 2014, 30, 488-495.

[19] L. Gremer, D. Schölzel, C. Schenk, E. Reinartz, J. Labahn, R. B. G. Ravelli, M. Tusche, C. Lopez-Iglesias, W. Hoyer, H. Heise, et al., Science (80-. ). 2017, 358, 116 119.

[20] M. A. Wälti, F. Ravotti, H. Arai, C. G. Glabe, J. S. Wall, A. Böckmann, P. Güntert, B. H. Meier, R. Riek, Proc. Natl. Acad. Sci. U. S. A. 2016, 113, E4976-E4984.

[21] R. Sarroukh, E. Goormaghtigh, J. M. Ruysschaert, V. Raussens, Biochim. Biophys. Acta - Biomembr. 2013, 1828, 2328-2338.

[22] J. Szczerbiński, J. B. Metternich, G. Goubert, R. Zenobi, Small 2020, 16, 1905197.

[23] Z. C. Zeng, H. Wang, P. Johns, G. V. Hartland, Z. D. Schultz, J. Phys. Chem. C 2017, $121,11623-11631$.

[24] T. Schmid, L. Opilik, C. Blum, R. Zenobi, Angew. Chemie Int. Ed. 2013, 52, 59405954.

[25] R. Sarroukh, E. Cerf, S. Derclaye, Y. F. Dufrêne, E. Goormaghtigh, J. M. Ruysschaert, V. Raussens, Cell. Mol. Life Sci. 2011, 68, 1429-1438.

[26] E. Cerf, R. Sarroukh, S. Tamamizu-Kato, L. Breydo, S. Derclayes, Y. F. Dufrênes, V. Narayanaswami, E. Goormaghtigh, J. M. Ruysschaert, V. Raussens, Biochem. J. 2009, $421,415-423$.

[27] J. C. Stroud, C. Liu, P. K. Teng, D. Eisenberg, Proc. Natl. Acad. Sci. U. S. A. 2012, 109, 7717-7722.

[28] S. Chimon, M. A. Shaibat, C. R. Jones, D. C. Calero, B. Aizezi, Y. Ishii, Nat. Struct. Mol. Biol. 2007, 14, 1157-1164.

[29] M. Törnquist, T. C. T. Michaels, K. Sanagavarapu, X. Yang, G. Meisl, S. I. A. Cohen, T. P. J. Knowles, S. Linse, Chem. Commun. 2018, 54, 8667-8684.

[30] M. Tornquist, R. Cukalevski, U. Weininger, G. Meisl, T. P. J. Knowles, T. Leiding, A. Malmendal, M. Akke, S. Linse, Proc. Natl. Acad. Sci. U. S. A. 2020, 117, $11265-$ 11273. 

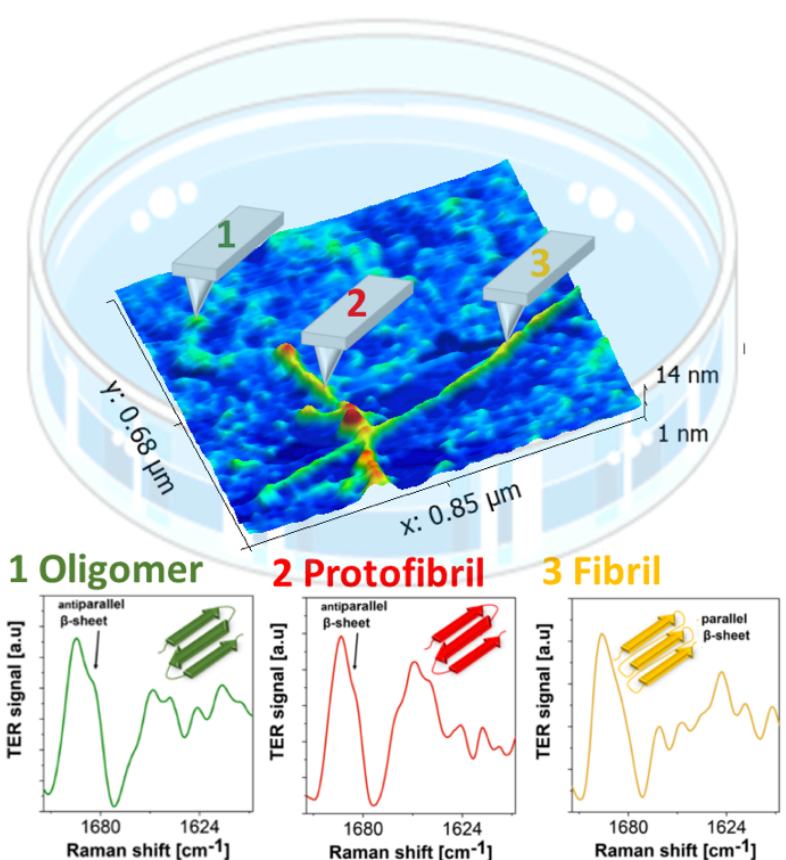

Tip-enhanced Raman spectroscopic (TERS) mapping in liquid proved to be an efficient tool in an investigation into the neurodegenerative peptides secondary structure. The high heat capacity of liquid increased the capability of TERS, allowing an observation of the conformational transition from the antiparallel arrangement of $\beta$-sheets in protofibrils to parallel in fibrils, at the single aggregates level. 